\title{
Grid and Cloud Computing: Opportunities for Integration with the Next Generation Network
}

\author{
Thomas Rings • Geoff Caryer • Julian Gallop • \\ Jens Grabowski • Tatiana Kovacikova • \\ Stephan Schulz • Ian Stokes-Rees
}

Received: 7 November 2008 / Accepted: 13 August 2009 / Published online: 28 August 2009

(C) Springer Science + Business Media B.V. 2009

\begin{abstract}
Carrier-grade networks of the future are currently being standardized and designed under the umbrella name of Next Generation Network (NGN). The goal of NGN is to provide a more flexible network infrastructure that supports not just data and voice traffic routing, but also higher level services and interfaces for thirdparty enhancements. Within this paper, opportunities to integrate grid and cloud computing
\end{abstract}

T. Rings $(\varangle)$ · G. Caryer · J. Gallop · J. Grabowski ·

Institute of Computer Science,

University of Göttingen, Goldschmidtstr. 7,

37077 Göttingen, Germany

e-mail: rings@cs.uni-goettingen.de

G. Caryer

e-mail: geoff@tmconsult.co.uk

J. Gallop

e-mail: julian.gallop@stfc.ac.uk

J. Grabowski

e-mail: grabowski@cs.uni-goettingen.de

T. Kovacikova

University of Zilina, Zilina, Slovakia

e-mail: tatiana.kovacikova@fri.uniza.sk

S. Schulz $\cdot$ I. Stokes-Rees

European Telecommunications Standards Institute,

Sofia Antipolis, France

S. Schulz

e-mail: stephan.schulz@etsi.org

I. Stokes-Rees

e-mail: ijstokes@spmetric.com strategies and standards into NGN are considered. The importance of standardized interfaces and interoperability testing demanded by carrier-grade networks are discussed. Finally, a proposal how the testing methods developed at the European Telecommunications Standards Institute (ETSI) can be applied to improve the quality of standards and implementations is presented.

Keywords Interoperability $\cdot$ Next generation network $\cdot$ NGN $\cdot$ Grid $\cdot$ Cloud $\cdot$ Standards . Testing $\cdot$ ETSI

\section{Introduction}

Carrier-grade networks form the global communications infrastructure that support millions of phone calls each day and, even more importantly, the massive global data transfers, predominantly resulting from the Internet. These carrier-grade networks are operated by hundreds of companies often deployed on top of physical infrastructure (cabling and switches). These infrastructures are usually not owned by the network operator but have to follow different regulations in each country and likely traverse several billing domains at any given point-to-point connection.

This globally integrated system operates with extremely low down time and transparently to the end users. This has been achieved through 
decades of development and standardization of interfaces, a process which has now become well established in the telecommunications industry. The latest evolution of the global communications networks, the Next Generation Network (NGN), is designed to support converged fixed and wireless networks carrying both voice and data traffic. Furthermore, these future networks incorporate a richer set of features to provide more services to customers, and hence increased revenue opportunities for the network providers. Increased flexibility around network-level services has also opened the door to third party services built on top of the NGN infrastructure.

With increasing interest in grid computing over the past several years and more recently, cloud computing, a major question that needs to be answered is how these technologies, concepts, and capabilities can be incorporated into NGN. Grid and cloud computing systems would benefit from enhanced capabilities of NGN, the global reach of existing communications networks, and the stability of carrier-grade networks.

Interoperability has been one of the key contributors to widespread commercial success of technologies used in the telecommunications sector, due to the interconnected nature of networks, and the plethora of network operators. Interoperability fosters diversity as well as competition in a market. Vendors can achieve interoperability of their products only if they agree and implement a common set of open standards. The value of standardization has also been recognized by the grid community, and is predominantly championed by the Open Grid Forum (OGF) [50] for grid-specific standards. Standardization, however, does not necessarily lead to interoperability. Standards have to be engineered for interoperability. Similarly, implementations of standards have to be assessed for their interoperability with other implementations and have to demonstrate that they follow these standards. At the standardization level, this can be facilitated with the availability of open and validated test specifications.

In this article, the accomplishments of the work completed by the Technical Committee GRID (TC GRID) of the European Telecommunications Standards Institute (ETSI) are reported. This work includes studies about the state of grid comput- ing, existing standards, and any gaps or overlaps that would need to be addressed for a cohesive carrier-grade grid computing environment to be provided by a network operator. The standards landscape around grid computing, the tension between grid standards and operational grids are discussed. In addition, the ETSI approach towards interoperability testing, standards evaluation, and the preparation of ETSI interoperability events, the so-called Plugtests ${ }^{\mathrm{TM}}$ [11] are presented. The interoperability events are used to compare various implementations in a controlled setting and can provide detailed feedback on the quality of a standard based on the level of interoperability that is achieved. In the longer term, ETSI plans to establish standards that will support the convergence between NGN, grid, and cloud computing environments.

The article is structured as follows: the standards landscape for grid, cloud and NGN domains are presented in Section 2. Their convergence is described in Section 3. In Section 4, the interoperability between grid systems is assessed. In Section 5, ETSI's approach to grid testing is introduced. Finally, we conclude with a summary and an outlook in Section 6.

\section{Standards Landscape for Grid, Cloud and NGN Domains}

The wide range of organizations involved with one or more of grid, cloud, and NGN technology each have their own priorities. Where operational systems have been designed or deployed, this range of priorities has resulted in competing architectures and interfaces. Although NGN does not yet exist as an integrated global telecommunications platform, there is a coordinated effort to develop the suite of standards to cover a high level NGN architecture [14]. In contrast, grid computing offers a few high level conceptual models, typically using the hour-glass middleware imagery. This envisages a wide range of high level applications connected to a wide range of heterogeneous low level resources via a limited number of intermediate standard interfaces. In addition, there are a few concrete architectural models for grid infrastructures [26, 47]. These concrete models have 
a distinct disconnection: either they present an architecture which is not or only partially implemented in any operational grid, or an architecture which describes a particular grid infrastructure with limited references to standards or interfaces. In the cloud domain, there is currently a prevalence of independent services with minimal interest in interoperability or consideration of standards. While this is in the process of changing, there is currently no sufficient activity in this area to report on.

While the original motivation for grid computing originated with large scientific collaborations, it is now established that the same new technology and perspective on distributed computing is applicable in many domains. The Networked European Software and Services InitiativeGrid (NESSI-Grid) review considered the impact of grid technology on business IT infrastructure [45], while several projects have considered the application of grid computing to eHealth (see case study in Section 4.1). The recent popularity of cloud computing also demonstrates the industry benefits of shared, distributed computing infrastructure.

\subsection{Differentiating Cloud Computing and Grid Computing}

It is important to distinguish grid computing from cloud computing. Grid computing has a longer history and has primarily been adopted by public sector compute or data intensive user groups. This community has a pressing need for large scale federated computing systems, and the development of standards has been in support of this.

Cloud computing, by contrast, originated in the private sector where virtualization technology and large data centers have been turned into the foundation for products and services to be resold. This section will help clarify the difference between the two. Subsequent sections will focus primarily on grid computing, as cloud computing still lacks any substantive standards or possibilities for interoperation, making any discussion around cloud computing and the telecommunications industry purely speculative.

The grid concept has a complementary but independent relationship to the concept of cloud computing. The similarities are that both aim to provide access to a large computing (CPU) or storage (disk) resource. Beyond that, a cloud utilizes virtualization to provide a uniform interface to a dynamically scalable underlying resource, with the intention that the virtualization layer conceals physical heterogeneity, geographical distribution, and faults. Current cloud environments only provide direct support for single user or single organization access, and current models typically have a high cost to integrate computing, data, or network transfers from outside of the cloud. This model suits environments where computing and data resource needs can be isolated to a single location and rapid scaling (up or down) of computing, network, and data availability are important. Pricing models are variations on normalized CPU-hours, GB/day storage, and MB network $\mathrm{I} / \mathrm{O}$, or are based on a cloud product that can be licensed and used with local physical resources.

In contrast, grid computing aims to provide a standard set of services and software that enable the collaborative sharing of federated and geographically distributed computing and storage resources. It provides a security framework for identifying inter-organizational parties (both human and electronic), managing data access and movement, and utilization of remote computing resources.

Cloud computing offers a solution to the problem of organizations that need resources (computing, storage, or network bandwidth) either quickly or with a highly dynamic level of demand. Operating in steady state at or near full capacity, cloud computing is still more expensive than direct ownership of computing resources, even if these are co-located in a shared data center. Cloud computing, at the present time, also only offers relatively bare bones systems on top of which a user or organization needs to deploy and manage their applications and data.

Grid computing addresses different issues around federated interoperation of computing facilities, security, shared data management, application deployment, system monitoring, and application or job execution. Grid computing can benefit from the development of cloud computing by harnessing new commercially available computing and storage resources, and by deploying 
cloud technology on grid-enabled resources to improve the management and reliability of those resources via the virtualization layer.

Cloud computing can benefit from grid concepts by integrating standard interfaces, federated access control, and distributed resource sharing. The current state of the art favors cloud computing for single organization commercial applications that can be deployed in their entirety onto a cloud environment. The dynamic provisioning of storage, computing power, and network bandwidth allows rapid scaling for intensive utilization either directly by the organization or by the public via Internet-based interfaces.

Grid technology continues to dominate public sector scientific computing environments due to the collaborative nature of this work and the need to manage existing data sets and computing resources across organizational boundaries. The more advanced state of interface standardization within grid technology allows some degree of choice between various software and hardware systems. Deploying data and applications into a cloud environment, however, limits an organization to a single cloud provider or requires duplicated effort to repeat the deployment process for additional cloud environments.

\subsection{Development and Adoption of Grid Standards}

Grid computing is a concept, not a product, a solution, or a single global network akin to the Internet. This concept is most succinctly communicated by I. Foster, C. Kesselman, and S. Tuecke in "The Anatomy of the Grid" [25]. The grid concept is realized through numerous grid (and grid-related) projects, (open-source) software, international collaborations, physical infrastructure, and operational grids. While the various aspects of the grid computing concept are still undergoing evaluation (standards, software, hardware configurations, and human organizational issues), it is difficult to form a single grid infrastructure or even grid architecture. Given the inherent collaborative and interoperable promise of grid computing, this is a major obstacle to its adoption in wider domains such as the business and private sector. In this section, we review the current state of grid standardization and standards adoption, considering standards bodies, de facto standards, formal architectures, and informal or ad hoc architectures.

The OGF [50] was formed by many of the original advocates and adopters of grid technology with the goal of identifying requirements for the various aspects of grid computing and then to develop standards that would allow the implementation of interoperable grid systems. Other standardization bodies and industry fora such as the Organization for the Advancement of Structured Information Standards (OASIS) [52], the World Wide Web Consortium (W3C) [67], the Internet Engineering Task Force (IETF) [36], and recently ETSI's TC GRID have also directly or indirectly participated in the process of establishing standards that are central to the realization of a standards-based interoperable grid environment.

There are some major grid projects that do aim to implement standards-based systems, such as the Globus Alliance [59] which develops the Globus Toolkit [22, 32], a low-level open source software toolkit used for building grid systems and applications. The Uniform Interface to Computing Resources (UNICORE) forum [63], based in Germany, and the Open Middleware Infrastructure Institute (OMII) for Europe [48] provide software environments that are standards conformant and cover a significant portion of the required components to realize an operational grid. The UNICORE software [57, 62] is used by the Distributed European Infrastructure for Supercomputing Applications (DEISA) [8], and also by the German Grid Initiative (D-Grid) [30].

In contrast, several large national and international grid infrastructures consist predominantly of custom-made software developed without reference to standards. These include the lightweight middleware for grid computing (gLite) software distribution [31, 38], developed by the Enabling Grids for E-sciencE (EGEE) project (primarily to support of the Worldwide Large Hadron Collider Computing Grid (WLCG)), the Open Science Grid (OSG) [51] and TeraGrid [58] projects in the US, Grid Operation System (GOS) [64, 68] the software developed by the China National Grid (CNGrid) [6], and Fura, a commercial product developed by Grid Systems [34]. 
The numerous grid research projects also play an important role in establishing the grid value chain. For example, within the European Union (EU)-funded Sixth Framework Programme (FP6) from 2002 to 2006, a number of research projects were funded in the area of advanced grid technologies, systems and services. These projects initiated collaborations between dozens of universities, research institutes, and large and small companies from across Europe to muster the critical mass of experience and resources necessary to stress test system interoperability, standards, and implementations of standards. Ongoing FP6projects and projects started in the Seventh Framework Programme (FP7) build on the results of FP6 and broaden it to encompass software services over the Internet.

An Eurescom report about business opportunities for telecom operators in the grid market concluded that telecom operators are bound to become key players in the grid value chain, as they provide connectivity and own computing resources [21]. Moreover, they have established customer relationships and accounting/billing experience, which is essential for business grids. Several major players including American Telephone \& Telegraph Corporation (AT\&T), British Telecommunications (BT), Deutsche Telekom (DT), France Telecom (FT) and Telefonica of Spain continue to invest resources required for measuring the market potential of grids and clouds.

To enable NGN support for grid and cloud services, it is necessary to identify the requirements of these services and to select existing standards, or develop new standards that ensure interoperability.

\subsection{Standard Grid Models}

Grid models are either explicitly stated or implicitly defined in a particular implementation. As a minimum, all grid models address security, networking, computing resources, storage resources, and information systems. How these areas are brought together, and what services, systems, and sub-systems provide a specific capability or interface, form the grid model and act as the basis for any standardization effort. The
Globus project proposed the Open Grid Service Architecture (OGSA) in 2002 [24], later refined in 2006 [26]. This model presents a grid as a Service Oriented Architecture (SOA).

To discuss grid infrastructure in a telecoms context, ETSI TC GRID has a developed a working model [15]. This can be depicted as a layering of services which can be utilized independently or together. In Fig. 1, these are grouped by the type of services they deliver. The lowest level represents the foundation of the infrastructure: networking, storage, computing power, and preexisting software applications. These are wrapped and presented as software services. The next layer represents services that are central to the operation of the grid, while the outer layer provides user-focused services.

These services are utilized by consumers, customers and providers. The consumer models the individual or organization using a grid services. The customer models the entity responsible for contracting the grid services, and pays for usage by consumers they have authorized. The provider models the entity providing grid services.

\subsection{The Architecture of NGN}

The NGN [46] is a global initiative from the telecoms industry using standards developed by ETSI and the International Telecommunication

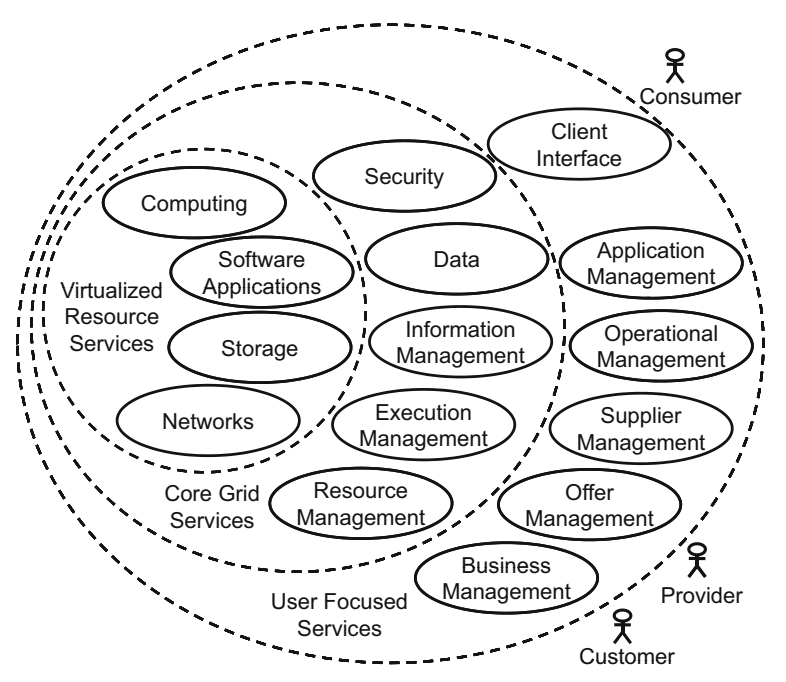

Fig. 1 ETSI conceptual model of a grid and associated roles [15] 
Union-Telecommunication Standardization Sector (ITU-T) [35]. This involves also members of ETSI TC Telecoms and Internet converged Services and Protocols for Advanced Network (TISPAN) that include telecom operators such as British Telecom, German Telecom, and France Telecom.

Like with other telecommunications technologies, such as ISDN or GSM, NGN standards intent to achieve interoperability. Thus, they shall enable equipment manufacturers to develop equipment that performs one or more NGN functions and that can interoperate with other manufacturer's equipment and service providers. The goal is to construct an NGN that supports the range of multimedia services that they wish to offer and that should interoperate with other networks. Based on proposals from members, ETSI and the ITU-T develop NGN standards to provide a consistent kit of building blocks, which allow manufacturers and service providers flexibility without compromising interoperability.

NGN is being designed to provide interoperable, inter-domain all IP-based network standards with enhanced multimedia capabilities. There is a global effort within the industry to transition to NGN and re-develop existing services to leverage NGN capabilities. Architecturally, servicerelated functions are independent from underlying transport-related technologies. As such, NGN offers unrestricted access by users to different service providers. Independently from the access technology, the NGN standards support mobility, nomadicity and multiple services. These include voice telephony, data services, multimedia services, virtual private networks, public network computing, and unified messaging [7]. The NGN design has aimed to support a dynamic architecture and can, therefore, accommodate new services as they are identified.

The NGN functional architecture that is defined by TISPAN [14] identifies two NGN layers. These are the Service Layer and the IP-based Transport Layer as shown in Fig. 2. Both layers are composed from subsystems which are specified as a set of functional entities and related interfaces.

IP-connectivity is provided to NGN user equipment (UE) by the transport layer, under the control of the Network Attachment Subsystem

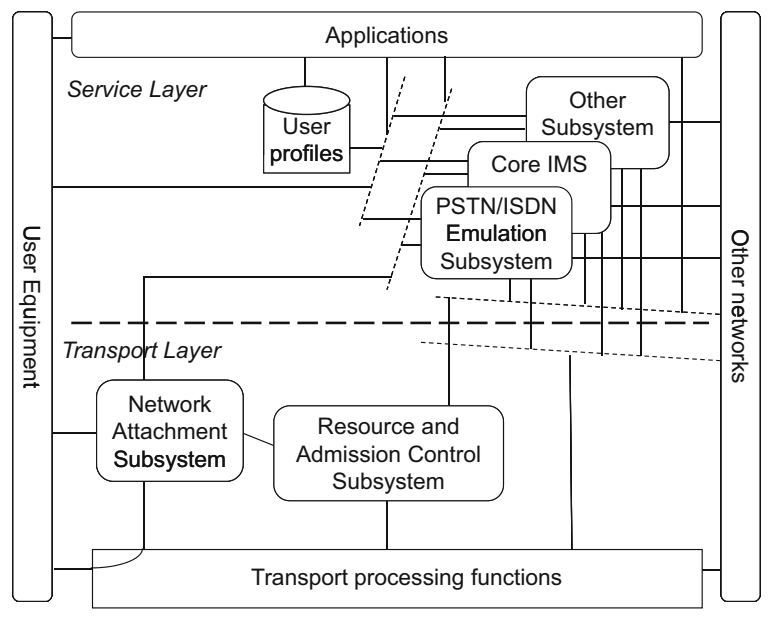

Fig. 2 TISPAN NGN overall architecture

(NASS) and the Resource and Admission Control Subsystem (RACS). These subsystems hide the transport technology that is used in access and core networks below the IP layer.

The service layer comprises the following subsystems:

- PSTN/ISDN Emulation Subsystem (PES): PES supports the emulation of PSTN/ISDN services through residential gateways or access gateways for legacy terminals connected to the NGN.

- Core Internet Protocol (IP) Multimedia Subsystem (IMS): IMS supports the provision of Session Initiated Protocol (SIP)-based services.

- Other subsystems, e.g. Internet Protocol Television (IPTV) dedicated subsystem) and applications.

- Common components for accessing applications, charging functions, user profile management, security management, routing databases that are used by several subsystems.

This subsystem-oriented architecture enables the addition of subsystems to cover new demands and service classes. In addition, it provides the ability to import and adapt subsystems defined by other standardization bodies. As depicted in Fig. 2, applications reside on the top of the service layer and can be accessed by other subsystems in this layer. TISPAN also defines an Application Server Function (ASF) [14] that can provide 
standalone services or value added services on top of a basic session.

\section{Convergence of NGN, Grid and Cloud Computing}

For telecom operators, the future lies in converging fixed, mobile and data services onto NGN. Historically, each service had its own platform with minimal interoperability. Integrating new services was made difficult by the lack of interoperability, resulting in high development and deployment costs, and consequently unattractive rates for the end users. Extending the NGN subsystem model to directly provide grid services, or at least provide mechanisms by which third parties can develop and deploy onto NGN grid services, would be the basis for significant new revenue potential and opportunities for a new era of networked applications and services.

With advances in commodity computer components in terms of speed, cost, and reliability, many parts of NGN can utilize commercial-offthe-shelf (COTS) hardware, rather than high cost specialized chips, switches, and associated hardware. This opens a second avenue for the integration of NGN with cloud computing approaches which would allow a network operator to virtualize various NGN subsystems, thus providing dynamic scalability, load-balancing, and fault tolerance. Past studies [28] have already identified the potential for cost savings if the current vertically siloed infrastructure, provisioned to handle peak loads which may be two or three times higher than the average load, could be re-deployed onto a more efficient shared physical infrastructure. With expertise in the domain of managing virtualized servers for NGN operations in large data centers, it is a small step for network operators to consider partitioning their virtualized server platforms and making these available as cloud computing services in a manner similar to the cloud services offered by Amazon [1, 29]. Telecom operators are increasingly considering SOA in order to:

- decouple applications via middleware from IT server/storage/network resources,
- flexibly compose new services using standards-based technologies and protocols,

- reuse architectural components to lower costs, and time-to-revenue.

There are features of grid technology that can directly benefit from different aspects of NGN by addressing control and sharing between heterogeneous system components. These are, for example, resource optimization and billing, high performance content rendering and encoding in the service layer, and co-allocation and cross optimization of network resources and grid resources (computer, storage). In summary, there are four possible scenarios:

1. Grid-enabled NGN application

2. NGN subsystems offering grid and cloud services

3. Combining grid and networking resources in a new architecture

4. Grid and cloud technology for implementing NGN functionality

In the first scenario, as depicted in Fig. 3, a gridenabled NGN application is deployed as an application server. The NGN is already designed to support a wide range of Application Servers and is capable of incorporating additional types developed by other groups. These application servers are available via a standard interface. The gridenabled application server would access interfaces from the Core IMS sub-system. In this scenario, the NGN could provide IP connectivity, autho-

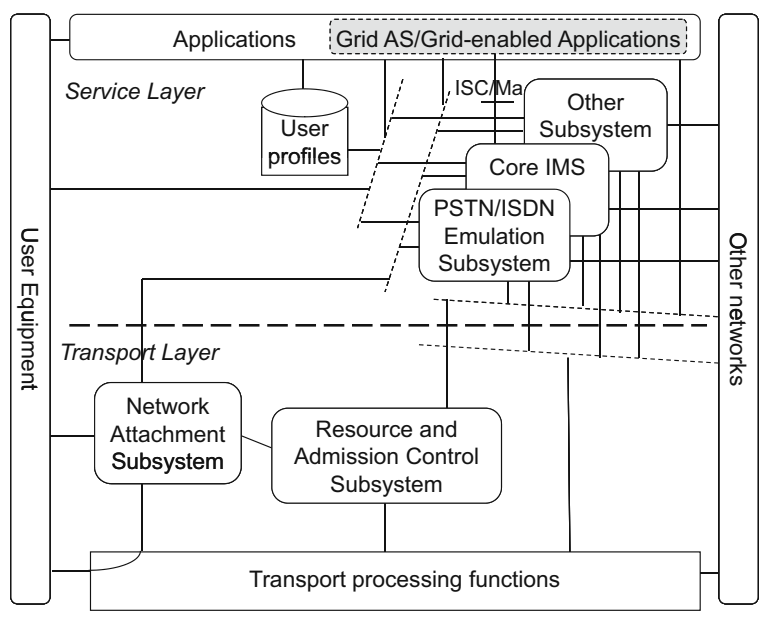

Fig. 3 Grid-enabled NGN application 


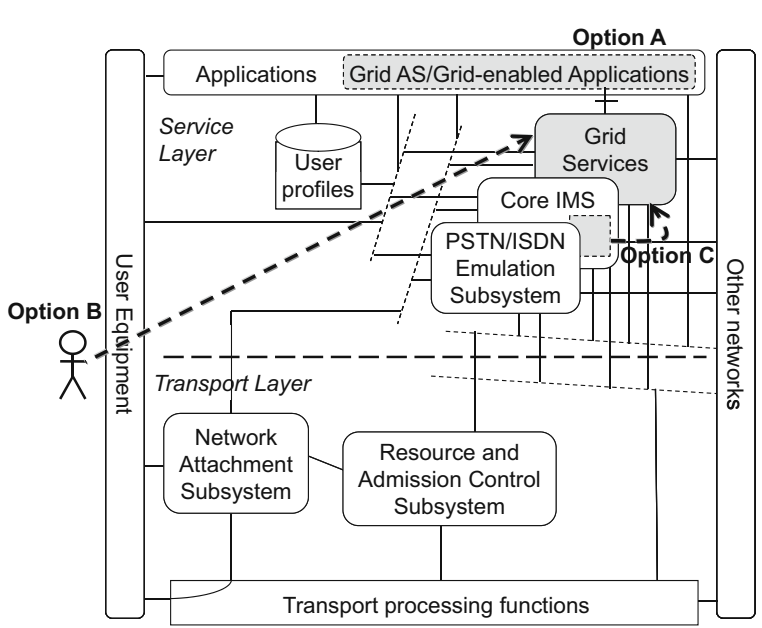

Fig. 4 NGN subsystems offering grid and cloud services

rization, security, Quality of Service (QoS), and charging.

In the second scenario, as depicted in Fig. 4, a new NGN subsystem is added to the NGN Service layer to support the provisioning of grid or cloud services. This Grid Services Subsystem (GSS) gives access to virtualized grid-enabled cloud resources, through a new service interface to the virtual resources and grid services. Such an integration of a GSS presents three different options:

(A) Grid-enabled applications as defined in the first scenario. The GSS interfaces would provide common grid functionality and resource access, thereby accelerating development of higher-level applications.

(B) Direct access by end-user applications, providing them with resources managed by the GSS in the same way that non-IMS IPTV services are offered.

(C) Updating other subsystems to leverage new capabilities offered by the GSS. This option requires the current NGN service layer subsystems to be enhanced to support grid services in the same way that IMS based IPTV services are offered.

In the third scenario "Combining grid and networking resources in a new architecture" a separate grid service manages shared resources such as computing power, network and storage. This would enable the assignment of resources to the grid or the NGN in a flexible, generic way.

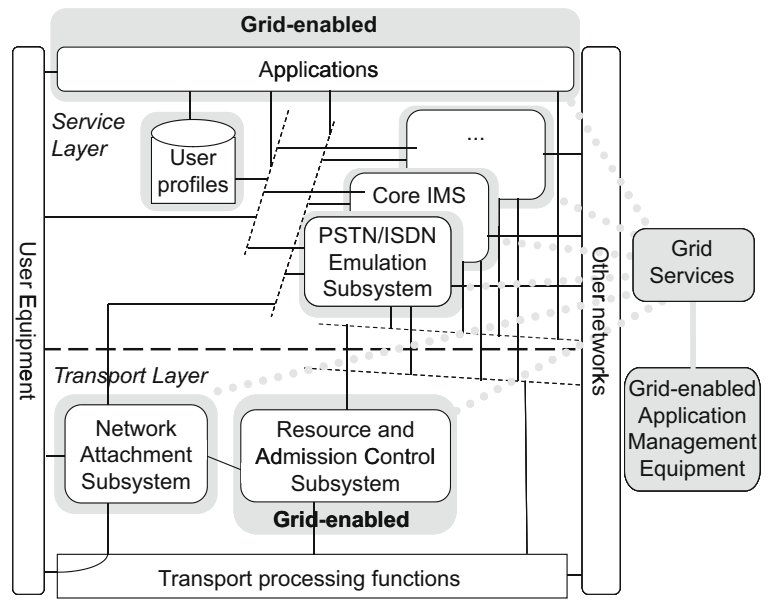

Fig. 5 Grid and cloud technology for implementing NGN functionality

The fourth scenario "Grid and cloud technology for implementing NGN functionality" enhances the entire NGN architecture with capabilities for harnessing virtualized cloud resources and grid-enabled services, as depicted in Fig. 5. This is the most disruptive and ambitious scenario, where logical NGN functions and entire NGN subsystems are refined in light of grid and cloud capabilities. This allows the optimization of the resources used by these functions and provides greater flexibility in the deployment and operation of various NGN subsystems.

As part of the ETSI TC GRID activities around NGN, grid, and cloud computing, these scenarios have all been documented in greater detail in [18].

\section{Grid Interoperability}

The focus of the ETSI TC GRID interoperability study was to consider areas where interoperability between grid and cloud infrastructures is possible either in theory or in practice. As the cloud computing domain is dominated by provider-specific interfaces, suitable to the single provider model of current cloud services, it was assessed that there is currently no significant opportunity to discuss interoperability in this area. Furthermore, there are no established standard interfaces applicable specifically to cloud computing, although some efforts have started to establish these. In contrast, there are a range of standards covering grid computing. This section considers those standards, 
the associated implementations, and the resulting state of interoperability.

\subsection{Case Studies: Integrated Emergency \\ Management and eHealth}

An important case study is Integrated Emergency Management (IEM), which clearly demonstrates the value and need for interoperability between conventional network services and enhanced distributed applications. IEM involves setting up an environment in which the contributions of multiple agencies can be planned and coordinated in a coherent way [61]. The European Access to Knowledge through the Grid in a mobile World (Akogrimo) project [40] demonstrated such a system in 2007, as described in a preparatory paper issued in 2006 [5]. IEM typically involves planning for emergencies, response when an emergency occurs, and a recovery period. In the response phase, this application is characterized by stringent QoS requirements in the presence of difficult conditions: resilience to broken connections; a requirement to cross conventional organizational boundaries; a need to be able to trust people from multiple organizations in the field; a need to optimize the brokering of a range of expert and specialist resources; and detecting and managing context changes.

Another important and illustrative application area is eHealth [19]. Within this application area are many different potential scenarios, but two that we mention here are Computerized Decision Support (CDS) and Patient Monitoring (PM). In one example of CDS, a medical research study called CREDO [27] highlights the needs of patients with medical conditions that must be served by many medical professionals, each offering different services, which need to form an effective whole. In one example, there were over 200 services and 65 critical decision points requiring support. Screening, diagnosis and planning involve the use of data and computational resources on an on-demand basis that require QoS negotiation. Authentication and security of patient data is critical. PM illustrates other problems, which were addressed in another Akogrimo demonstrator [40]. Patient monitoring away from a medical setting has the potential to use sensors and a wireless network connection, delivering measurements to an evaluation point. Requirements include mobile grid services, secure communication, occasional bursts of intensive computing, initiation of conference calls between available, relevant experts and tailoring the visualization to the results of discovery of visual displays.

Identity management, security, and reliability are critical and multi-agency heterogeneous resources are inherent in the way health and emergency resources are managed. Authentication of a professional not previously involved with the particular case must be efficient. The need for interoperability is critical since these scenarios involve multiple conventional organizations.

\subsection{Standards Adoption}

Figure 6 illustrates the standards that are in use in several popular grid software packages. These are dominated by OGF standards associated

\begin{tabular}{|c|c|c|c|c|c|c|}
\hline & Standard & Unicore 6 & GT 4 & GLite & GOS v3.2 & Fura \\
\hline \multirow[t]{5}{*}{ Security } & $\mathrm{X} .509$ & $\sqrt{ }$ & $\sqrt{ }$ & $\sqrt{ }$ & $\checkmark$ & $\checkmark$ \\
\hline & Security Assertion Markup Language (SAML) & $\sqrt{ }$ & $\checkmark$ & $\checkmark$ & $\checkmark$ & \\
\hline & extensible Access Control Markup Language (XACML) & $\checkmark$ & $\checkmark$ & $\checkmark$ & & \\
\hline & Virtual Organization Membership Service (VOMS) & $\checkmark$ & $\checkmark$ & $\checkmark$ & & \\
\hline & WS-Security (Transport Level Security (TLS)) & $\sqrt{ }$ & $\sqrt{ }$ & $\checkmark$ & $\sqrt{ }$ & $\checkmark$ \\
\hline \multirow[t]{3}{*}{ Execution } & Job Submission Description Language (JSDL) & $\checkmark$ & $\sqrt{ }$ & $\checkmark$ & $\checkmark$ & \\
\hline & OGSA-BES (Basic Execution Service) & $\checkmark$ & & $\sqrt{ }$ & $\checkmark$ & \\
\hline & Distributed Resource Management Application API (DRMAA) & $\checkmark$ & $\sqrt{ }$ & $\checkmark$ & & \\
\hline \multirow[t]{2}{*}{ Data } & OGSA-BytelO & $\checkmark$ & & & & \\
\hline & GridFTP (defacto) & & $\checkmark$ & $\checkmark$ & & \\
\hline \multirow[t]{3}{*}{ Information } & Web Service Resource Framework (WSRF) & $\sqrt{ }$ & $\sqrt{ }$ & $\sqrt{ }$ & & \\
\hline & OGSA-RUS (Resource Usage Service) & $\checkmark$ & & & $\checkmark$ & \\
\hline & OGSA-UR (Usage Record) & $\checkmark$ & & $\checkmark$ & $\checkmark$ & \\
\hline
\end{tabular}

Fig. 6 Comparison of implemented standards in grid middleware 
with OGSA and WSRF. Clearly the Public-KeyInfrastructure (PKI) X.509 certificate system has found wide-spread adoption in all grid domains. The table also shows that the OGSA-Basic Execution Service (OGSA-BES), OGSA-Resource Usage Service (OGSA-RUS), and OGSA-Usage Records (OGSA-UR) standards have the broadest adoption amongst the middleware under consideration.

One aspect that is not captured by this figure is the number of grid infrastructures that utilize very few standards. In the US, TeraGrid and OSG both are dominated by custom-made software, or packages distributed through the Virtual Data Toolkit (VDT) [60]. In these cases, the only visible standards are GridFTP and the X.509 identity system. Even authorization by X.509 certificates is handled in quite different ways on different grids. In actuality, no operational grid infrastructures rely exclusively (or even predominantly) on grid standards, but instead use a patchwork of custommade and third-party software packages, expecting "interoperable" sites to be running the same version of the software in order to maximize the possibility of successful interoperability.

It is also essential to note that higher level grid applications will rely on underlying grid middleware services in such a way that interoperability is not always possible, even when two implementations support the same underlying set of standards. This also occurs because of incomplete or patchwork standards environment.

\subsection{Current Approaches to Interoperability}

Two approaches are pursued and partially applied to address grid middleware interoperability: gateway/adapter and standardization. The first approach introduces a gateway between two systems. This is a short term solution, since a change in one implementation may break the adapter and thus the interoperability.

The gateway approach has been used by [65] to achieve interoperability between the EGEE grid infrastructure based on the gLite middleware and the CNGrid based on the GOS middleware. In this case, the gateway approach has been applied because gLite and GOS implement very different architectures. A gateway has been applied for their job and data management service to submit jobs and access data by the other middleware. For example, when a job is submitted, a $J o b$ Description Language (JDL) file required by gLite is translated into a Job Submission Description Language (JSDL) file expected by GOS and vice versa.

Standardization as a basis for interoperability has been analyzed by [41]. This work focused on interoperability of job submissions between gLite and UNICORE since the other core services of each have custom-made implementations. However, UNICORE and gLite adopt common open standards such as OGSA-BES [23] and the JSDL [3] specification which means that the same client can invoke operations based on these standards within UNICORE or gLite. In addition to these common interfaces, it was found that both implementations needed to handle X.509 user identity tokens and permissions in a similar way. OMII-UK [49] also includes projects which focus on implementing standards-based grid services, such as GridSAM, the JSDL application repository and OMII-SAGA.

Finally, the OGF has formed a specific community group to consider interoperability issues, galled Grid Interoperation Now (GIN) [33]. This group reviews the current state of grid interoperability and works towards improving interoperability [53]. GIN provides feedback to OGF standards bodies and leaders of grid software projects in cases where interoperability is not possible.

\subsection{Standardization Gaps}

Building confidence in interoperability relies on well scoped, well-specified and achievable standards. Gaps and overlaps within and between standards cause problems which have to be solved by infrastructure providers and application developers. To this end an analysis of gaps and overlaps was undertaken by ETSI TC GRID. The methodology adopted was to identify broadly applicable themes for gap analysis, develop a number of case studies as a mechanism to identify gaps, bring together gaps, overlaps and issues based on these themes and, on more general architectural con- 
siderations, make proposals for the resolution of gaps, overlaps and issues.

Gaps and overlaps are likely to be encountered in several different situations:

- The presence of complex applications involving the composition of services horizontallya combination of grid services-and vertically-grid services making use of multiple networks, one of which could be NGN.

- The relationship between a grid and the underlying network.

- Diversity allowed within an individual standard which could lead to incompatible implementations.

- The use of dynamic working, which exposes problems with standards which are designed with a static situation in mind.

- Transition to a new version of a specification. When considering the whole landscape of many standards, effective transition becomes a complex problem.

- Lack of implementations.

\subsection{Classification of Gaps}

In our study, the gaps and overlaps were classified into four broad themes: security, Service Level Agreement (SLA) and QoS, charging, and service discovery. The identified gaps mentioned in the next paragraphs can be addressed in the short term for specific applications and testing frameworks through policy-based constraints, while others will require longer term standards.

\subsubsection{Security}

Security includes authentication, authorization, protection and trust. The problems of authentication of NGN User Equipment (UE) are similar to the ones of user, host and service identities in grids. Both areas (NGN and grid) lack standards to guide development. Significant standard gaps exist around the issue of authorization in the grid domain. The standards related to the operation of a security infrastructure are limited. In addition, a lack of standards regarding the use of groups, roles and attributes as part of a dynamic Virtual Organization (VO) exists.

\subsubsection{SLA and $Q o S$}

For certain critical types of distributed infrastructure, there is a need for effective and timely alternative action when the terms of the SLA are violated in order to maintain a temporarily adequate level of service. The structure of SLA service description terms is defined, but the names and semantics of them are defined outside the SLA standards. These descriptions have been considered for computational resources but not as yet for the relationship with networks (for instance, with NGN). Multi-provider applications will present problems for SLA establishment and monitoring.

\subsubsection{Charging}

Charging includes the activity of collecting usage data from resources. Existing solutions do not allow situations where service providers are discovered and selected dynamically. Providing a single bill in a multiple provider application requires further information to be delivered. There is an overlap between the structure of grid usage records and NGN Charging Detail Records, which will prove to be a problem when NGN becomes one of the supporting technologies for grids.

\subsubsection{Service Discovery}

Existing solutions for service discovery such as Universal Description, Discovery and Integration (UDDI) have proved to be inadequate because of its static nature. A standard is required for grid service registry that can support VO-style authentication, a high level of dynamism and the inclusion of service state. There is significant overlap of the capabilities provided by various griddomain directory services and service discovery mechanisms. Thus, there is significant scope to analyze the requirements of these different systems and work towards a common grid directory service standard. Existing solutions for service discovery do not work well for device identification, which are needed for situations such as sensor networks. 


\subsection{High-Level Issues Contributing to Gaps}

Several gaps are not due to issues around a particular technology area but come from human processes, composition of services or architectural layers, and the life-cycle of complex systems.

One such deficiency occurs where implementations have not yet caught up with the OGF and OASIS standards. Production grids, which are now extensive, adopted grid software which predated the move to Web Services and because of the initial success were slow to move on. This appears to be changing, because Production Grid Infrastructure Working Group (PGI-WG) has recently been formed within OGF in order to solve specific problems that the production grids are meeting when they adopt current OGF standards.

Other gaps result from considering the relationship between a grid and the underlying network. For instance OGF specifies information which may be used for charging. Even though, this is also covered by IETF Internet standards as well as NGN standards from ETSI. There is a need for the standardization process to enable these different mechanisms to work at all levels.

The design of complex applications involves the composition of services. Any area of cross-cutting concerns is thereby affected by these compositions. The strongest example of this is the security infrastructure, which needs to be consistent and comprehensive in the presence of a composed application. Security policies need to be shared and merged in a predictable way. This is an area which is not currently covered by grid standards. SLAs are also affected where composed services need to be negotiated effectively building on the existing SLAs of constituent services. Similarly, charging needs to take the composition of services into account. In general, there is little standards coverage of charging models for grid services.

Within each individual grid standard, there is often room for variation, either intentionally to allow flexibility, or unintentionally. This can inhibit interoperability between implementations, if different subsets of the variations are chosen. This potential for incompatibility needs to be corrected either by restricting variation in standards or by incorporating protocols for service characteristic enquiry and negotiation.
Complexity due to dynamic operational environments introduces gaps. Behavior during transition from one environment to another is generally not discussed: for example in mobile services where the network characteristics such as bandwidth or QoS may change during a single session. Although existing Grid specifications envisage some variation of user requirements and workloads, they generally do not accommodate variations in an active session.

Many grid protocol and data standards have not considered the standard life-cycle and environments where multiple standards are in use concurrently. Accommodating version negotiation and compatibility will introduce problems. For example, this is known to occur where Web Service (WS)-Agreement [2] references a different version of WS-Addressing from the one used by most current implementations. Explicit versioning needs to be introduced. This will become critical as complex applications running across several grids come into use.

\section{Testing for Interoperability}

Conformance and interoperability testing involves checking that implementations follow their specifications and that they provide the intended functionality. In the following we use the term standard instead of specification, because we consider such a specification to be standardized and published by an organization like OGF, W3C or ETSI. In this section, we describe the details of conformance and interoperability testing, discuss related work on grid testing and describe the ETSI process for conformance and interoperability test development. Finally, we present an example that shows how the ETSI process can be used to define interoperability tests for grid systems. The example is based on the OGF OGSA-BES standard [23].

\subsection{Conformance vs. Interoperability Testing}

At ETSI, implementations of standards can be formally tested using conformance testing, interoperability testing, or interoperability testing with conformance checking [13]. The three approaches 
are illustrated in Fig. 7. Each approach has benefits and limitations.

Conformance testing is functional black-box testing of one Implementation Under Test (IUT) where the IUT shows its conformity to a standard. The IUT is normally embedded within a System Under Test (SUT). The SUT is a testing environment that contains the IUT and possibly emulates parts of the system the IUT has to interact with to provide its service to its user. However, even passing a conformance test does not automatically prove that the IUT is interoperable with other systems implementing the same standard. The reason for this is that standards are often not precise enough; they contain implementation options and requirement specifications leave space for interpretation.

Interoperability testing demonstrates that two or more Equipments Under Test (EUTs) together provide the end-to-end functionality described or implied by a standard. In this setting, an EUT is considered to be a complete system that may consist of several soft- and hardware components. The EUTs inter-operate via an abstract Means of Communication (MoC) which is not subject of the interoperability test. Therefore, the SUT for interoperability testing only includes the EUTs and not the MoC. However, there exists the hidden as-
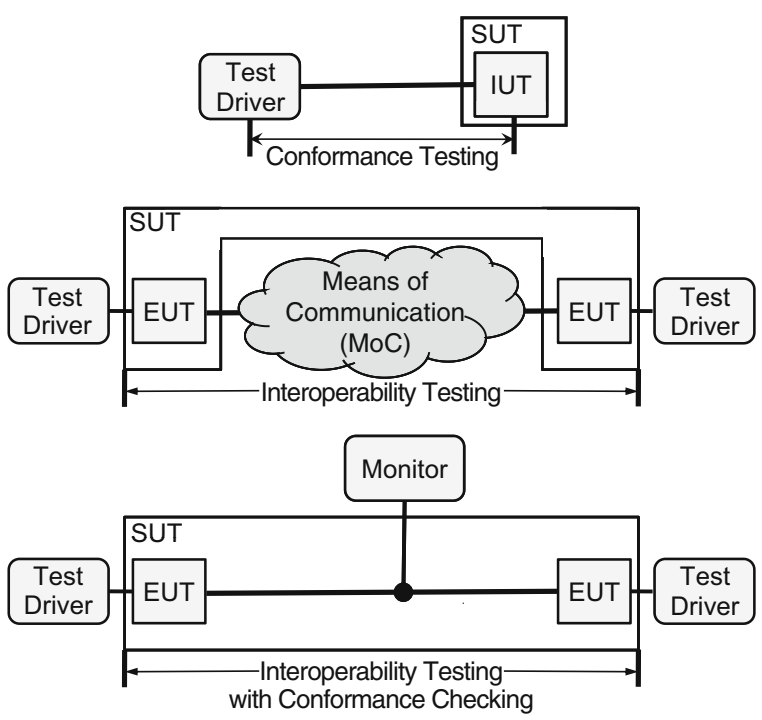

Fig. 7 Three approaches to testing sumption that the communication services necessary for the interoperation of the involved EUTs have been tested for conformance before the interoperability test takes place. Unfortunately, practice has shown that a lot of interoperability problems are related to conformance problems of these communication interfaces.

For this reason, ETSI advocates interoperability testing with conformance checking, a hybrid of the two approaches described above, as interoperability testing approach. This third approach extends end-to-end interoperability testing with the monitoring of the communication among the EUTs. The monitor is used to check the conformance of the EUTs with the relevant protocol specifications within the SUT during the interoperability test.

The ETSI experience with applying this hybrid approach in Plugtests ${ }^{\mathrm{TM}}$ has been that in a number of cases end-to-end interoperability has been observed even though EUTs did not communicate according to underlying standards. Although this approach is not a replacement for conformance testing, it gives an insight into the conformance to a standard of EUTs involved in the interoperability test.

\subsection{Related Work on Grid Testing}

An approach of testing grid application workflows based on the grid middleware Globus Toolkit 4 is outlined in [54]. This work focuses on conformance testing adopting the international ISO/IEC multi-part standard 9646 OSI Conformance Testing Methodology and Framework [37] for test specification and implementation using the Testing and Test Control Notation (TTCN-3) [20, 66]. A case study demonstrates that the test concepts of TTCN-3 and the remote communication mechanisms provided by TTCN-3 run-time environments facilitate very well the distributed testing in grid environments.

The eInfrastructure for Testing, Integration and Configuration of Software (ETICS) project [9] provides a build and test system by means of reliable grid software and industry-standard best practices. ETICS is used for the build and integration process of gLite by EGEE. Examples for ETICS-based implementations of EGEE are the 
IPv6 compliance analysis of gLite code and the distributed IPv6 experimental testbed for testing the IPv6 version of the Berkely Database Information Index (BDII). In addition, EGEE applied the ETICS system to build some of its high-level services, such as GridWay. Furthermore, the ongoing ETICS 2 project endeavors to support the widespread adoption of grid technologies.

OGF has done some work on test specifications for ByteIO [43] and GridRPC [44]. These test specifications are informal test descriptions that mainly follow a conformance oriented, unit testing approach rather than end-to-end functionality testing from an ETSI point of view.

\subsection{The ETSI Process for Test Development}

The development of test specifications at ETSI $[12,56]$ is a process that goes through multiple steps as illustrated in Fig. 8. These steps can be understood as different levels of abstraction that bridge the large gap between a base standard or profile thereof and a final conformance or interoperability test specification.

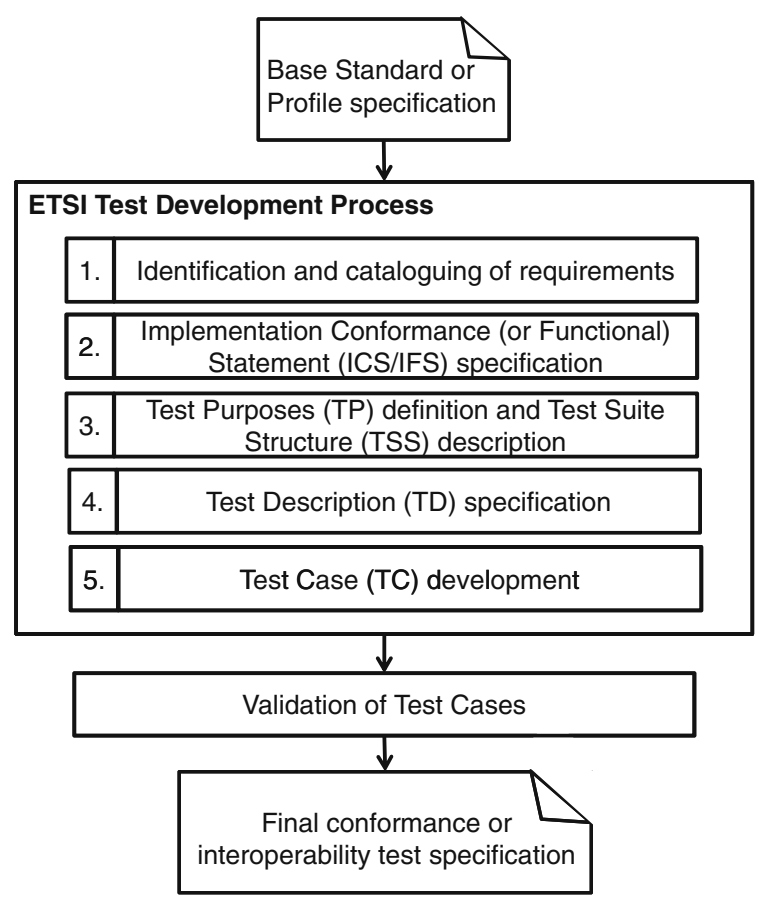

Fig. 8 ETSI process for test development
In step 1, requirements are identified from relevant base specifications or profiles thereof. A requirement is a specific behavior of the IUT, i.e., a series of stimuli to and expected outputs from the IUT, that can be assessed by means of a test. Requirements may be published in a requirements catalogue. Then, in step 2, the Implementation Conformance (or Functional) Statement (ICS/IFS) is specified. This step is essentially a high level check list of features and capabilities supported by the IUT. The ICS/IFS can be used to quickly identify if two implementations of the same standard have the potential to inter-operate. In the next step (step 3), Test Purposes (TPs) are specified for the identified requirements and a logical grouping of the TPs, the Test Suite Structure (TSS) is defined. If a requirement can be assessed using a given form of testing then a test purpose specifies verdict criteria for a test. After that, in step 4, for each TP an informal Test Description (TD) is developed. In step 5, either TP- or TD-based Test Cases (TCs) are specified.

The final step includes the validation of the TCs and is normally not done by ETSI. The validation ensures that the TCs are correctly specified. It may be done by executing the TCs at an interoperability event or by running TCs by means of conformance test tool against a number of different implementations of a given standard. Problems detected during the validation should be reported to ETSI and may lead to changes in the ETSI TC specifications. The validated TCs form the final interoperability or conformance test specification.

\subsection{Example: Grid Interoperability Test Specification for OGSA-BES CreateActivity}

In this section, the ETSI test specification development process is exemplified by using an excerpt of the requirements identified in the OGF OGSA-BES standard [23]. OGSA-BES is a good candidate for interoperability testing, because interoperation between OGSA-BES implementations is defined in the standard and OGSA-BES is not only implemented by the grid systems presented in Fig. 6, but also by other grid-, cluster-, and cloud-environments such as Microsoft HPC Server [42], GridSam [39], and BES++ [4, 55]. 


\subsubsection{OGSA-BES-A Short Introduction}

OGSA-BES is an OGF standard that specifies a Web Service for initiation, monitor, and control of computational activity requests from a $B a$ sic Execution Service (BES) client to a server. Activities in an OGSA-BES context are described with JSDL [3] and can be, for example, UNIX or Windows processes, Web Services, or parallel programs within a defined environment. A BES server executes accepted activities on appropriate computational resources. These resources can be a single computer, a cluster managed by a resource manager, a Web Service hosting environment, or another BES server.

\subsubsection{Test Configuration}

Prior to starting test development, an appropriate test configuration for the interoperability test with conformance checking has to be defined. For this, we need to relate the service oriented view of the grid infrastructure (Fig. 1) to a view of physical elements. This is done in the test configuration shown in Fig. 9 which allows testing interoperability between OGSA-BES-client and OGSA-BESserver implementations.

In this figure, we assume that one EUT includes a grid middleware like gLite, Globus Toolkit 4, or UNICORE that implements user focused ser-

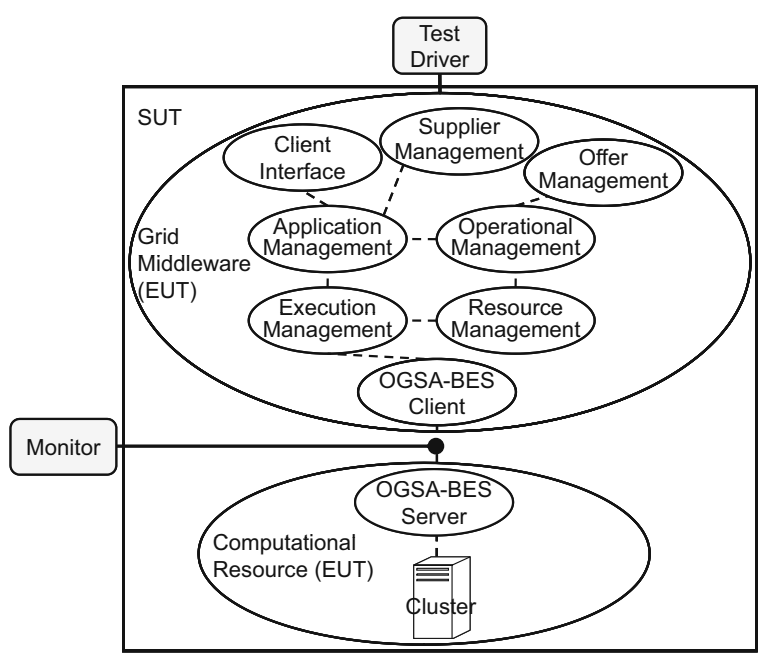

Fig. 9 Test configuration reflecting physical entities as well as services involved in creating a BES activity vices, core grid services, and an OGSA-BES-client service. The second EUT is a computational resource like a single computer or cluster managed by an OGSA-BES-server implementation. During the interoperability test, a monitor component checks the conformance of the client-server communication to the standard referenced in [23].

\subsubsection{Requirement Extraction}

The first step in the development of ETSI test specifications is to analyze and extract requirements from a base standard or a profile thereof. Figure 10 shows the specification of the CreateActivity operation as an excerpt from OGSABES [23]. It is used for requesting the creation of new activities. For the CreateActivity operation, inputs, output, and also the handling of faults are specified. The CreateActivity operation expects a JSDL input file that includes a job description element and describes a single activity. If the activity is created successfully, the operation returns the activity identifier. In addition, the specification defines several faults that may occur. For example, the consumer requesting the creation may not be authorized to create activities, the BES service may currently not accept new activities, a feature described in the JSDL may not be supported by the BES implementation, or an element in the request message may not be recognized.

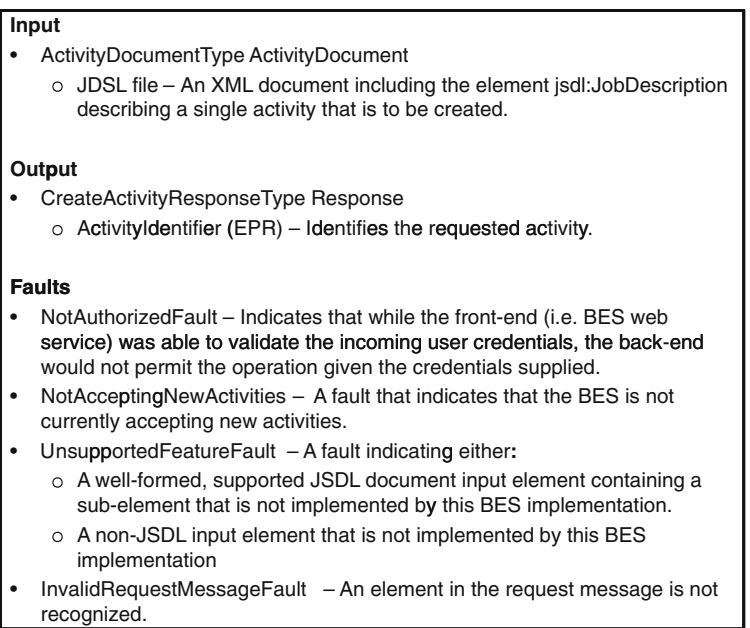

Fig. 10 CreateActivity specification (Excerpt from OGSA-BES) 
Determining the granularity of requirements to capture is not always an easy task. The CreateActivity operation description contains arguably more than a single requirement. It covers the handling of normal behavior as well as the handling of exceptional or invalid behavior. However, instead of isolating these behaviors during requirement extraction, we retain them in a single, compound requirement and isolate them in the next step, the test purpose specification.

\subsubsection{Test Purpose Specification}

The next step in the ETSI test development process is the specification of TPs. For this, ETSI has developed TPLan [56], a special notation for describing TPs. An example of a TPLan description is shown in Fig. 11. TPLan is based on natural language and can be used to enforce structured and consistent writing of TPs.

A TP associated with an interoperability test with conformance checking has to cover either one of two aspects: (1) identification of the endto-end functionality to be tested, or (2) the conformance requirements that can be assessed during the end-to-end test.

Figure 11 presents a TP for the second aspect, i.e., for a conformance test which assesses the correct implementation of a valid BES CreateActivity operation invocation. Additional test purposes have also been specified for testing the error handling as described in Fig. 10.

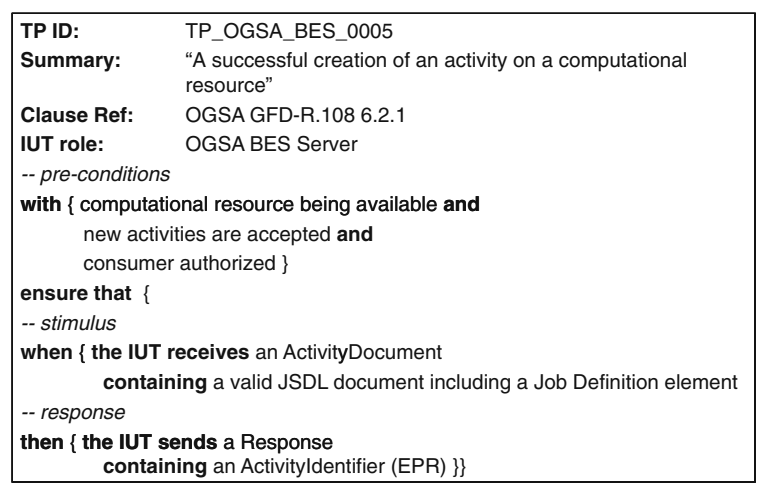

Fig. 11 Test purpose for a successful BES activity creation

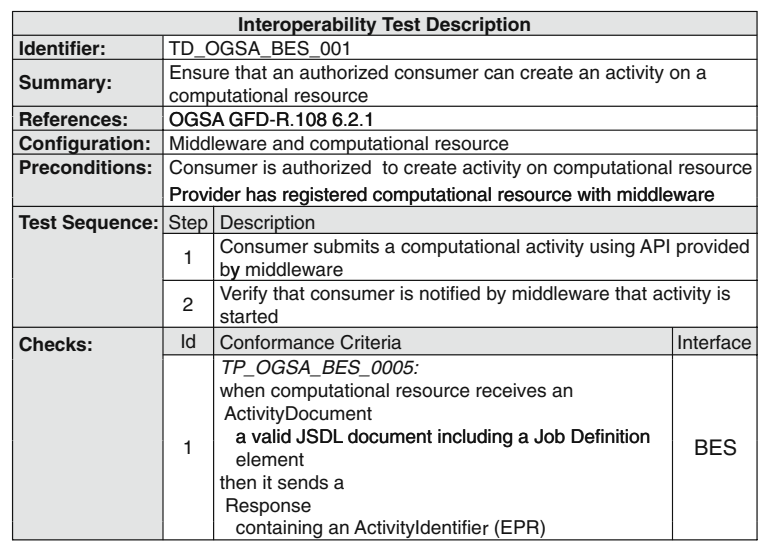

Fig. 12 Test description for a successful BES activity creation

\subsubsection{Specification of Test Descriptions}

After defining conformance TPs, we have to select end-to-end functionalities that include the TPs and to specify a complete test description for each functionality. An example for such a test description is shown in Fig. 12.

The test sequence is written in terms of external actors and their ability to interact and observe the services provided by the entire grid infrastructure, i.e., end-to-end behavior. Based on its success, we derive a test verdict reflecting the interoperability of all EUTs in a test.

The test description also includes a list of all relevant conformance TPs that can be assessed by monitoring the EUT-communication during the end-to-end test. The compliance of test execution traces to all of these TPs forms another test verdict, the so-called communication verdict. Since end-to-end functionality usually encompasses the exchange of multiple messages, each interoperability test usually covers a number of different conformance TPs at the same time. In our example (Fig. 12), we have limited the assessment to only one conformance TP.

\subsection{ETSI Plugtests ${ }^{\mathrm{TM}}$}

Test descriptions like the one shown in Fig. 12 are often validated at interoperability events like, for example, the ETSI Plugtests ${ }^{\mathrm{TM}}$ [11]. Currently, ETSI organizes around 15 Plugtests $^{\mathrm{TM}}$ per year 
also in corporation with other organizations and fora. Plugtests ${ }^{\mathrm{TM}}$ are open for attendance to everyone. They are used to assess or demonstrate the maturity of a given technology as well as to validate standards, e.g., for IMS core networks, HighDefinition Multimedia Interface (HDMI), IPv6, Radio-Frequency IDentification (RFID), IPTV, power line transmissions, intelligent transport systems, etc.

ETSI has also been organizing grid Plugtests ${ }^{\mathrm{TM}}$ for the past five years [10]. Recently, its technical format has been changed from a grid programming contest on a fixed, worldwide grid infrastructure into the assessment of a common interface for application deployment on a variety of different grid and cloud infrastructures based on ETSI Grid Component Model (GCM) standards [16, 17]. Results and findings of this Plugtests ${ }^{\mathrm{TM}}$ are fed back to the ETSI TC GRID and will be used for the improvement of existing and the creation of new standards in this domain.

\section{Summary and Outlook}

Telecom operators are expecting that grid enabled services can improve their internal network operation as well as enrich the services they offer to their customers. For this, interoperability between grid technology and telecom networks has to be achieved. ETSI and its TC GRID have a key role to play in establishing priorities, standards, and testing mechanisms.

Several possible scenarios for converging grid and cloud technology with NGN, discussing the advantages and disadvantages of each have been considered in this article. A review of key standards and standards development organizations highlights the successes, as well as the shortcomings indicated by many custom-made grid infrastructures. Cloud computing has recently become a popular area, but presently lacks standards or perspectives for interoperability, although there are signs this is slowly changing. ETSI intends to continue standardizing software protocols and interfaces relevant to NGN and adopting grid and cloud computing technology into the global telecommunications network. The annual Plugtests ${ }^{\mathrm{TM}}$ have potential to grow into a much broader evaluation of commercial grid technology and standards.

Interoperability between systems can only be achieved when there are clear standards for interfaces and an environment that supports multiple implementations of architectural components. The lack of a widely agreed-upon grid architecture, encompassing software, hardware, and services, impedes the development of a consistent set of standards.

The telecom industry will gain valuable experience with third party services and sub-systems offering advanced functionality with the roll-out of NGN. We expect that this roll-out will lead to increased efforts to develop interoperating grid, cloud, and telecom systems.

Acknowledgements The authors would like to thank all reviewers of this paper for their helpful contributions as well as the ETSI TC GRID for their guidance and support. We would also like to thank the European Commission for the funding provided under the contract SA/ETSI/ENTR/000/2006-10 which has made this article possible.

\section{References}

1. Amazon Web Services, LLC: (Online; http://aws. amazon.com/ fetched on 13-04-09)

2. Andrieux, A., Czajkowski, K., Dan, A., Keahey, K., Ludwig, H., Nakata, T., Pruyne, J., Rofrano, J., Tuecke, S., Xu, M.: Web Services Agreement Specification (WS-Agreement), GFD-R-P.107. Open Grid Forum (2007)

3. Anjomshoaa, A., Brisard, F., Drescher, M., Fellows, D., Ly, A., McGough, S., Pulsipher, D., Savva, A.: Job Submission Description Language (JSDL) Specification, Version 1.0, GFD-R.136. Open Grid Forum (2008)

4. BES++ Project Homepage: (Online; http://bespp. sourceforge.net/ fetched on 13-04-09)

5. Briscombe, N., Palmer, D., Ntuba, M., Bertram, S., Boniface, M.: Enabling integrated emergency management: reaping the Akogrimo benefits. Tech. rep., IT Innovation Centre, School of Electronics and Computer Science, University of Southampton. (Online; http://eprints.ecs.soton.ac.uk/14197/1/Akogrimo_white Paper_DisasterCrisisMgmt_v1-1.pdf fetched on 14-04-09) (2006)

6. China National Grid: (Online; http://www.cngrid.org fetched on 13-04-09)

7. Crimi, J.C.: Next Generation Network (NGN) Services. Telcordia Technologies, White Paper (2003) 
8. Distributed European Infrastructure for Supercomputing Applications: (Online; http://www.deisa.eu/ fetched on 13-04-09)

9. eInfrastructure for Testing, Integration and Configuration of Software (ETICS): (Online; http://www. eu-etics.org fetched on 13-04-09)

10. ETSI: History of Events. (Online; http://www.etsi.org/ WebSite/OurServices/Plugtests/History.aspx fetched on 28-07-09)

11. ETSI: Plugtests ${ }^{\mathrm{TM}}$ interop events. (Online; http:// www.etsi.org/plugtests fetched on 13-04-09)

12. ETSI: ETSI ES 202 568: Methods for Testing and Specification (MTS); Internet Protocol Testing (IPT); Testing: Methodology and Framework. European Telecommunications Standards Institute (ETSI), Sophia-Antipolis, France (2004)

13. ETSI: ETSI ES 202 237: Methods for Testing and Specification (MTS); Internet Protocol Testing (IPT); Generic approach to interoperability testing. European Telecommunications Standards Institute (ETSI), Sophia-Antipolis, France (2007)

14. ETSI: ETSI ES 282 001: Telecommunications and Internet converged Services and Protocols for Advanced Networking (TISPAN); NGN Functional Architecture. European Telecommunications Standards Institute (ETSI), Sophia-Antipolis, France (2008)

15. ETSI: ETSI TR 102 659-1 V1.1.1: GRID; Study of ICT Grid interoperability gaps; Part 1: Inventory of ICT Stakeholders. European Telecommunications Standards Institute (ETSI), Sophia-Antipolis, France (2008)

16. ETSI: ETSI TS 102827 V1.1.1: Grid Component Model (GCM); GCM Interoperability Deployment. European Telecommunications Standards Institute (ETSI), Sophia-Antipolis, France (2008)

17. ETSI: ETSI TS 102828 V1.1.1: Grid Component Model (GCM); GCM Applications Description. European Telecommunications Standards Institute (ETSI), Sophia-Antipolis, France (2008)

18. ETSI: ETSI TR 102 767: Grid services and telecom networks; Architectural option. Tech. rep., European Telecommunications Standards Institute (ETSI), Sophia-Antipolis, France (2009)

19. ETSI eHEALTH Technical Body: (Online; http:// http://portal.etsi.org/portal_common/home.asp?tbkey1= eHEALTH fetched on 13-04-09)

20. ETSI TC MTS: ETSI ES 201873 V3.2.1: The Testing and Test Control Notation version 3; Parts 1-8. European Telecommunications Standards Institute (ETSI), Sophia-Antipolis, France, also published as ITU-T Recommendation series Z.140 (2007)

21. EURESCOM: TelCo Grid - Business Opportunities for Telecom Operators in the Grid market. (Online; http://www.eurescom.de/public/projects/P1300-series/ P1349 fetched on 30-06-09) (not publicly available) (2004)

22. Foster, I.: Globus toolkit version 4: software for service-oriented systems. In: Jin, H., Reed, D.A., Jiang, W. (eds.) NPC. Lecture Notes in Computer Science, vol. 3779, pp. 2-13. Springer, New York (2005)
23. Foster, I., Grimshaw, A., Lane, P., Lee, W., Morgan, M., Newhouse, S., Pickles, S., Pulsipher, D., Smith, C., Theimer, M.: OGSA basic execution service version 1.0, GFD-R.108. Open Grid Forum (2008)

24. Foster, I., Kesselman, C., Nick, J., Tuecke, S.: The physiology of the grid: an open grid services architecture for distributed systems integration. http:// www.globus.org/alliance/publications/papers/ogsa.pdf (2002)

25. Foster, I., Kesselman, C., Tuecke, S.: The anatomy of the grid: enabling scalable virtual organizations. Int. J. High Perform. Comput. Appl. 15(3), 200 (2001)

26. Foster, I., Kishimoto, H., Savva, A., Berry, D., Djaoui, A., Grimshaw, A., Horn, B., Maciel, F., Siebenlist, F., Subramaniam, R., Treadwell, J., Reich, J.V.: The Open Grid Services Architecture, Version 1.5, GFD-I.080 (2006)

27. Fox, J., Patkar, V., Thomson, R.: Decision support for healthcare: the PROforma evidence base. Inform. Prim. Care 14, 49-54 (2006)

28. France Telecom R\&D: Grid computing: computer resources on demand. Tech. rep., France Telecom R\&D. (Online; http://www.orange.com/en_EN/innovation/ latest_news/hot_topics/tous_les_dossiers/att00009564/ ddm_200503uk.pdf fetched on 20-08-09) (2005)

29. Garfinkel, S.L.: An Evaluation of Amazon's Grid Computing Services: EC2, S3 and SQS. Tech. rep., Center for Research on Computation and Society, School for Engineering and Applied Sciences, Harvard University, Cambridge, MA (2007)

30. German Grid Initiative (D-Grid): (Online; http:// www.d-grid.de fetched on 13-04-09)

31. gLite: (Online; http://glite.web.cern.ch/glite/ fetched on 13-04-09)

32. Globus Toolkit: (Online; http://www.globus.org/ toolkit/ fetched on 13-04-09)

33. Grid Interoperation Now Community Group: (Online; http://forge.gridforum.org/sf/projects/gin fetched on 13-04-09)

34. Grid Systems S.A.: (Online; http://www.gridsystems. $\mathrm{com} /$ fetched on 13-04-09)

35. International Telecommunication UnionTelecommunication Standardization Sector (ITU-T): (Online; http://www.itu.int/ITU-T/ fetched on 13-04-09)

36. Internet Engineering Task Force: (Online; http://www.ietf.org/ fetched on 13-04-09)

37. ISO/IEC: Information technology-open systems interconnection-conformance testing methodology and framework. International ISO/IEC multipart standard No. 9646 (1994-1997)

38. Laure, E., Fisher, S.M., Frohner, A., Grandi, C., Kunszt, P., Krenek, A., Mulmo, O., Pacini, F., Prelz, F., White, J., Barroso, M., Buncic, P., Hemmer, F., Meglio, A.D., Edlund, A.: Programming the Grid with gLite. In: Jin, H., Reed, D.A., Jiang, W. (eds.) Computational Methods in Science and Technology, vol. 12(1), pp. 33-45. Scientific Publishers OWN (2006)

39. London e-Science Centre: GridSAM - grid job submission and monitoring web service. (Online; 
http://gridsam.sourceforge.net/2.1.3/index.html fetched on 13-04-09)

40. Loos, C.: E-health with mobile grids: The Akogrimo heart monitoring and emergency scenario. Akogrimo White Paper, (Online; http://www.akogrimo.org/ download/White_Papers_and_Publications/Akogrimo_ eHealth_white_paper_short_20060207.pdf fetched on 14-04-09) (2006)

41. Marzolla, M., Andreetto, P., Venturi, V., Ferraro, A., Memon, A.S., Memon, M.S., Tweddell, B., Riedel, M., Mallmann, D., Streit, A., van den Berghe, S., Li, V., Snelling, D.F., Stamou, K., Shah, Z.A., Hedman, F.: Open standards-based interoperability of job submission and management interfaces across the grid middleware platforms gLite and UNICORE. In: E-SCIENCE '07: Proceedings of the Third IEEE International Conference on e-Science and Grid Computing, pp. 592-601. IEEE Computer Society, Piscataway (2007)

42. Microsoft: Windows HPC Server 2008. (Online; http:// www. microsoft.com/hpc/en/us/developer-resources. aspx fetched on 13-04-09)

43. Morgan, M.: ByteIO OGSA WSRF Basic Profile Rendering 1.0, GFD-R-P.088. Open Grid Forum (2006)

44. Nakada, H., Matsuoka, S., Seymour, K., Dongarra, J., Lee, C., Casanova, H.: GridRPC: a Remote Procedure Call API for Grid Computing. Advanced Programming Models Research Group, GWD-I (Informational) (2002)

45. Networked European Software and Services Initiative: Business Grids Vision and Strategic Research Agenda v3.0. Online http://www.nessi-europe.com/ (2008)

46. Next Generation Networks: (Online; http://www.etsi. org/website/Technologies/NextGenerationNetworks. aspx fetched on 13-04-09)

47. NextGRID: (Online; http://www.nextgrid.org/ fetched on 13-04-09)

48. OMII-Europe: (Online; http://omii-europe.com/ fetched on 13-04-09)

49. OMII-UK: (Online; http://www.omii.ac.uk/ fetched on 13-04-09)

50. Open Grid Forum: (Online; http://www.ogf.org/ fetched on 13-04-09)

51. Open Science Grid: (Online; http://www. opensciencegrid.org/ fetched on 13-04-09)

52. Organization for the Advancement of Structured Information Standards (OASIS): (Online; http://www. oasis-open.org fetched on 13-04-09)

53. Riedel., M., Laure, E., Soddemann, T., Field, L., Casey, J., Litmaath, M., Baud, J.Ph., Koblitz, B.: Interoper- ation of world-wide production e-science infrastructures. Concurrency and Computation: Practice and Experience (2008)

54. Rings, T., Neukirchen, H., Grabowski, J.: Testing Grid application workflows using TTCN-3. In: International Conference on Software Testing Verification and Validation (ICST), pp. 210-219. IEEE Computer Society, Piscataway (2008)

55. Ruiz-Alvarez, A., Smith, C., Humphrey, M.: BES++: HPC profile open source $C$ implementation. In: 9th IEEE/ACM International Conference on Grid Computing, pp. 41-48 (2008)

56. Schulz, S., Wiles, A., Randall, S.: TPLan-a notation for expressing test purposes. In: TestCom/FATES. Lecture Notes in Computer Science, vol. 4581, pp. 292-304. Springer, New York (2007)

57. Streit, A., Erwin, D., Lippert, T., Mallmann, D., Menday, R., Rambadt, M., Riedel, M., Romberg, M., Schuller, B., Wieder, P.: UNICORE - From Project Results to Production Grids. CoRR (2005)

58. TeraGrid: (Online; http://www.teragrid.org/ fetched on 13-04-09)

59. The Globus Alliance: (Online; http://www.globus.org/ fetched on 13-04-09)

60. The Virtual Data Toolkit: (Online; http://vdt. cs.wisc.edu/ fetched on 13-04-09)

61. UK Cabinet Office: Civil contingencies act 2004: Emergency preparedness. (Online; http://www. cabinetoffice.gov.uk/ukresilience/preparedness . aspx fetched on 13-04-09) (2004)

62. UNICORE: (Online; http://www.unicore.eu/ fetched on 13-04-09)

63. UNICORE Forum e.V.: (Online; http://www. unicore.eu/forum/ fetched on 13-04-09)

64. VEGA GOS: (Online; http://vega.ict.ac.cn/gos/ en/index_en.htm fetched on 13-04-09)

65. Wang, Y., Scardaci, D., Yan, B., Huang, Y.: Interconnect EGEE and CNGRID e-Infrastructures through interoperability between gLite and GOS middlewares. In: eScience, pp. 553-560. IEEE Computer Society, Piscataway (2007)

66. Willcock, C., Deiß, T., Tobies, S., Keil, S., Engler, F., Schulz, S.: An Introduction to TTCN-3. Wiley, New York (2005)

67. World Wide Web Consortium (W3C): (Online; http://www.w3.org/ fetched on 13-04-09)

68. Zha, L., Li, W., Yu, H., Xie, X., Xiao, N., Xu, Z.: System software for China national Grid. In: Jin, H., Reed, D.A., Jiang, W. (eds.) NPC, Lecture Notes in Computer Science, vol. 3779, pp. 14-21. Springer, New York (2005) 